\title{
Patient handover and the opportunity for a pause, further enhancing the culture of patient safety
}

\author{
Martina Melvin ${ }^{1 *}$ and Fauzia Bano ${ }^{2}$ \\ ${ }^{1}$ Anaesthesia Specialist Registrar,Mater Misericordiae Hospital, Dublin, Ireland \\ ${ }^{2}$ Consultant Anaesthetists, Mater Misericordiae Hospital, Dublin, Ireland
}

\begin{abstract}
The handover of a patient in the post anaesthesia recovery unit from anaesthetist to recovery nurse is a brief and busy process. Certain elements of the patient history can be omitted. This loss of information affects patient safety.

Ten patient handovers were assessed, involving a different nurse and anaesthetist for each. Immediately after handover the nurse and anaesthetist completed a form asking them to recall patient details from the handover without referring to the medical notes. An independent observer then reviewed the medical notes and assessed if the information the nurse and anaesthetist recalled from handover corresponded with the medical notes. Data was analysed as percentage correlation of information.

A questionnaire was also distributed among the anaesthetists and recovery nurses, asking them if a handover checklist would aid with the handover process.

The highest correct correlations were patient age, co-morbidities, and drug allergies with 80\%, 80\% and $90 \%$. Anaesthetic technique, surgery performed and blood loss scored $70 \%$. There was only $50 \%$ correlation with analgesia and intravenous fluids given and $30 \%$ with ant-emetic given. $70 \%$ agreed a handover checklist would be helpful.

A handover checklist which provides both the nurse and anaesthetists with an opportunity for a pause is proposed to be incorporated into the handover process following this.
\end{abstract}

\section{Introduction}

Patient handover has been defined as 'the transfer of professional responsibility and accountability for some or all aspects of care for a patient, or group of patients, to another person or professional group on a temporary or permanent basis' [1].

Patients are in a vulnerable state in the post anaesthesia care room, both recovery nurses and anaesthetists are under 'time pressure' [2]. The patient's journey from theatre to recovery and then back to their original ward bed must not be delayed unnecessary, for to do so has implications on the general flow of the theatre lists. With this fast pace comes a risk of missing important information during handover of the patient $[1,3]$.

\section{Methods}

10 patient handovers were assessed. After the handover, the nurse and anaesthetist completed an information form asking them to recall patient details from the handover without referring to the medical notes. An independent observer then reviewed the medical notes and assessed if the information the nurse and anaesthetist recalled corresponded with the medical notes. Data was analysed as percentage correlation of information.

A questionnaire was also distributed asking them if an official handover checklist would aid handover.

\section{Results}

The information transferred with the highest correlations were patient age, co-morbidities, and drug allergies with $80 \%, 80 \%$ and $90 \%$ respectfully. Anaesthetic technique, surgery performed and blood loss scored $70 \%$ each.

There were only $50 \%$ correlation with analgesia and IV fluids, and $30 \%$ with ant-emetic given.70\% agreed a handover checklist would be helpful. The results show that there are gaps in patient handover.

The results were presented at a Departmental meeting. With $70 \%$ agreeing that an official handoverchecklist would be helpful, the various modes of communication available, verbal, written, electronic and combination were discussed. The plan going forward involves inclusion of a patient handover checklist into the Anaesthesia information management system once established. This system is becoming increasingly available in hospitals. It provides aspecialised form of electronic health record in which data is automatically collected and stored during the perioperative period [4].

A checklist with the necessary patient details would accompany the patient on transfer to the post anaesthesia care unit. The expectation is that the anaesthetist and recovery nurse would pause and review the checklist systematically thereby reducing the chances of information being omitted.

Correspondence to: Martina Melvin, Anaesthesia Specialist Registrar, Mater Misericordiae Hospital, 47 Cavalry House, Islandbridge, South Circular Road, Dublin, Tel: 0877860010. E-mail: melvin31@live.co.uk

Received: September 30, 2017; Accepted: October 20, 2017; Published: October 23,2017 


\section{Discussion}

The AAGBI safety guidelines on 'Immediate post Anaesthesia Recovery' 2013 and the Royal College of Anaesthetists 'Compendium of Audit Recipes' 2012 have clear standards set out regarding the information patient handover must include [2,5]. With familiarity amongst staff regarding the WHO surgical safety checklist [6], the proposed checklist for patient handover was favoured by most, as the appreciation for the impact on patient safety grows.

It is acknowledged, however, that with all the checklists accompanying patients throughout their journey in hospital, it sometimes becomes apparent that the focus is on checking boxes on the list, rather than focusing on the patient. The introduction of yet another checklist risks aggravating this further. That is why our checklist would serve as a handover aid, rather than a mandatory document that must be ticked off. The checklist provides the 'opportunity for a pause', between the anaesthetist and recovery nurse. This opportunity for a pause is a rarity in the busy anaesthesia recovery unit.

Patient handover serves as a fundamental part of the anaesthetist's work and is an important element ofcontinuity, quality, and safety in patient care $[7,8]$.

\section{References}

1. British Medical Association. Safe handover: safe patients. Guidance on clinical handover for clinicians and managers. London: BMA, 2004.

2. Membership of the Working Party:1, Whitaker Chair DK, Booth H, Clyburn P, HarropGriffiths W, et al. (2013) Immediate post-anaesthesia recovery 2013: Association of Anaesthetists of Great Britain and Ireland. Anaesthesia 68: 288-297. [Crossref]

3. post-anaesthesia_recovery_2013 [ accessed on 29th December 2016]

4. Catchpole K, Sellers R, Goldman A, McCulloch P, Hignett S (2010) Patient handovers within the hospital: translating knowledge from motor racing to healthcare. Quality Safety Health Care 19: 318-322.

5. Till A, Sall H, Wilkinson J (2014) Safe Handover: Safe Patients - The Electronic Handover System. BMJ Qual Improv Rep 2: u202926.w1359. [Crossref]

6. The Royal College of Anaesthetists. Raising the Standard: a compendium of audit recipes for continuous improvement in anaesthesia.

7. World Health Organisation surgical safety checklist and implemtation manual 2008.

8. Miller C (1998) Ensuring continuing care: styles and efficiency of the handover process. Aust J Adv Nurs 16: 23-27. [Crossref]

9. Kerr MP (2002) A qualitative study of shift handover practice and function from a socio-technical perspective. J Adv Nurs 37: 125-134. [Crossref]

Copyright: (C2017 Melvin M. This is an open-access article distributed under the terms of the Creative Commons Attribution License, which permits unrestricted use, distribution, and reproduction in any medium, provided the original author and source are credited. 